\title{
ABSORÇÃO DO OZÔNIO EM ÁGUA UTILIZANDO COLUNA DE PAREDE MOLHADA COM PROMOTOR DE PELÍCULA
}

\author{
C. F. FONSECA ${ }^{1}$ e A.C.S.C. TEIXEIRA ${ }^{1}$ \\ ${ }^{1}$ Universidade de São Paulo, Departamento de Engenharia Química \\ E-mail para contato:chrisfonseca@usp.br
}

\begin{abstract}
RESUMO - A ozonização tem sido considerada uma tecnologia promissora para tratamento de água e efluentes e no processamento de alimentos, cuja eficiência está diretamente relacionada à transferência de massa (TM) do gás para o líquido devido à baixa solubilidade do ozônio em água. Neste estudo, a solubilidade e o coeficiente global de TM (kLa) foram determinados utilizando uma coluna de parede molhada com promotor de película, sistema ainda não descrito na literatura para estudos ozônio/água. Experimentos foram realizados com gás e líquido em contracorrente a $20{ }^{\circ} \mathrm{C}$, para diferentes $\mathrm{pH}(3,5$ e 7) e concentrações de ozônio na fase gasosa, medindo-se a concentração de ozônio dissolvido no líquido em função do tempo. Os valores encontrados foram confrontados com a literatura e apresentaram um desvio relativo baixo para constante de Henry ( $2 \%$ e 5,6\%). Os valores obtidos de kd $(0,011$; 0,$016 ; 0,019 \mathrm{~min}^{-1}$ ) confirmaram uma maior decomposição em $\mathrm{pH}$ mais elevado $(\mathrm{pH} 7 ; 0,019$ $\min ^{-1}$ ). Os valores de kLa obtidos foram 0,$076 ; 0,091$ e $0,064 \mathrm{~min}^{-1}$ valores menores que os obtidos em colunas de bolhas. O estudo fornece dados importantes que podem contribuir para o entendimento de diferentes processos de ozonização empregando o sistema proposto.
\end{abstract}

\section{INTRODUÇÃO}

O ozônio tem sido usado na Europa como agente desinfetante no tratamento de água desde o começo do século XX e sua utilização tem crescido em diversas áreas, entre elas o tratamento de efluentes, bem como o processamento e desinfecção de alimentos (Cullen et al., 2009; Reinik et al., 2004). Uma das grandes vantagens do ozônio é o alto potencial como agente oxidante $(2,07 \mathrm{~V})$, quando comparado a outros agentes, como o cloro $(1,35 \mathrm{~V})$. Entretanto, o ozônio possui baixa solubilidade em meio aquoso se comparado aos outros oxidantes e, portanto, para distribuição do ozônio da fase gasosa para a fase líquida de maneira eficiente os tipos de sistemas de contato são de extrema importância e dependem de diversos fatores, tais como: vazão de líquido, vazão de gás, concentração de ozônio no gás e no líquido, características hidrodinâmicas e características físico-químicas da solução (Béltran, 2004). A solubilidade do ozônio na maioria das vezes é regida pela lei de Henry, em que a concentração de equilíbrio na fase líquida se relaciona com a concentração de ozônio na fase gasosa (Gottschalk et al., 2000), como mostra a Equação 1:

$$
H=\frac{\left[O_{3}\right]_{g}}{\left[O_{3}\right]_{l i q}^{*}}
$$

A solubilidade do ozônio em água tem sido estudada por diversos autores que obtiveram modelos empíricos válidos para determinados intervalos de temperatura e $\mathrm{pH}$, como apresentadas na Tabela 1 (Bin, 2006; Langrais et al., 1991; Morris, 1988; Quederni et al., 1987; Roth et al., 1981): 
Tabela 1 - Correlações para predição da solubilidade do ozônio em água

\begin{tabular}{ccc}
\hline Referências & Correlação & Aplicação \\
\hline Bin, 2006 & $\log _{10} H^{*}=6,5987-\frac{1752}{T}$ & $\begin{array}{c}\text { pH não informado } \\
\text { Temperatura: } 0 \text { a } 35^{\circ} \mathrm{C}\end{array}$ \\
\hline Langlais et al., 1991 & $H=e^{22,3-\left(\frac{4030}{T}\right)}$ & $\begin{array}{c}\text { pH não informado } \\
\text { Temperatura: } 0 \text { a } 35^{\circ} \mathrm{C}\end{array}$ \\
\hline Morris, 1988 & $\log _{10} S=3,302-0,013 T$ & $\begin{array}{c}\text { pH não informado } \\
\text { Temperatura: } 0 \text { a } 80{ }^{\circ} \mathrm{C}\end{array}$ \\
\hline Quederni et al., 1987 & $H=e^{\left(18,1-\frac{2846}{T}\right)}$ & $\begin{array}{c}\text { pH } 7 \\
\text { Temperatura: } 20 \text { a } 50{ }^{\circ} \mathrm{C}\end{array}$ \\
\hline Roth et al., 1981 & $H=3,84 \times 10^{7}\left[O H^{-}\right]^{0,035} e^{-\left(\frac{2428}{T}\right)}$ & $\begin{array}{c}\text { pH: } 0,65 \text { a } 10,2 \\
\text { Temperatura: } 3,5 \text { a } 60{ }^{\circ} \mathrm{C}\end{array}$ \\
\hline
\end{tabular}

$S$, razão de solubilidade (adimensional); $H^{*}$, Constante de Henry (adimensional); $H$, Constante de Henry (atm); $\left[\mathrm{OH}^{\top}\right]$, concentração de ions hidroxila (mol/L); T, temperatura $(\mathrm{K})$.

O ozônio pode reagir com diferentes substâncias segundo os mecanismos direto e indireto. A via indireta está relacionada com radicais e prevalece na presença de $\mathrm{OH}^{-}$, formando radicais hidroxila $\left({ }^{\circ} \mathrm{OH}\right)$, os quais não são seletivos e reagem rapidamente com outras espécies em solução. A via direta (ozônio molecular) é caracterizada por uma maior seletividade e por uma cinética lenta (Gottschalk et al., 2000), o que também depende da solubilidade do gás. Roth e Sullivan (1981) relacionam a constante de Henry ao pH e à temperatura nas condições estudadas (Tabela 1). O modelo apresentado por esses autores mostra que a solubilidade do ozônio decresce quando o $\mathrm{pH}$ aumenta e que a decomposição do ozônio é maior quanto maior o pH. Outros autores mencionados na Tabela 1 consideram como fator de influência somente a temperatura afirmando que quanto maior a temperatura menor a solubilidade do ozônio na água. Baseado nos valores da constante de Henry, o ozônio como dito anteriormente, é considerado um gás pouco solúvel em água. Nos processos de transferência de massa entre duas fases (gás-líquido), se o gás é pouco solúvel na fase líquida, a resistência da fase gasosa à transferência de massa entre as duas fases é desprezível quando comparada com a fase líquida, isto é, a resistência da fase líquida controla o processo. Quando o gás é altamente solúvel na fase líquida a resistência da fase gasosa controla o fenômeno de transferência de massa (Cremasco, 2002).

A geometria do sistema de contato é um parâmetro importante nos processos de ozonização, no entanto existem diversos estudos em relação a sistemas de coluna de bolhas e tanque de mistura (Mizuno et al., 2010; Sotelo et al., 1987). Em geral, os efeitos dos fatores físico-químicos e da fluidodinâmica do líquido e do gás não são facilmente calculados nesses sistemas (Rizzuti et al., 1976). Um sistema de fácil montagem e que permite estudar esses efeitos é a coluna de parede molhada (Danckwerts, 1970). Segundo Reinik et al. (2004) esse tipo de coluna tem a vantagem de ter a área interfacial conhecida e evitar a formação de espuma no sistema. Considera-se a área interfacial igual à geométrica, caso o escoamento seja um filme totalmente uniforme. A coluna consiste de um tubo circular, em suja superfície interna a água escoa, formando um filme. Pelo espaço no centro da coluna escoa a fase gás no sentido ascendente, o que configura um processo em contracorrente. Visando aumentar a irrigação da superfície em todo o comprimento e a renovação do filme Salvagnini e Taqueda (2004) estudaram um evaporador com um promotor de película, o qual consistia de uma malha metálica colocada rente à parede interna do evaporador. Os resultados indicaram que a utilização de um promotor de película aumentou a taxa de evaporação em até três vezes e que 
seu uso é apropriado em todas as operações em que a área interfacial é um fator importante, como nos processos de evaporação, absorção e destilação. Recentemente, a coluna com promotor de película foi utilizada para absorção de $\mathrm{CO}_{2}$ em soluções com aminas em escala de laboratório com objetivo de calcular parâmetros de transferência de massa para estas reações (Rodriguez-Flores et al., 2013)

Neste estudo, uma coluna de parede molhada com promotor de película foi utilizada para realizar experimentos de absorção de ozônio em água. O objetivo da utilização do filme foi melhorar a formação do filme e criar uma distribuição melhor ao longo da coluna evitando caminhos preferenciais, o que normalmente ocorre nas colunas de parede molhada sem o promotor de película. Experimentos preliminares foram realizados com água visando estudar a absorção sem reação para cálculo de parâmetros de transferência de massa, como coeficiente de transferência de massa $(\mathrm{kLa})$, a constante de Henry $(\mathrm{H})$ e o coeficiente de decomposição $(\mathrm{kd})$. A temperatura foi mantida constante, assim como as vazões de líquido e gás. A concentração de ozônio no gás e o pH foram variados.

\section{MATERIAL E MÉTODOS}

\subsection{Experimental}

Os experimentos de ozonização foram realizados em uma coluna de parede molhada com promotor de película que consiste de dois tubos de vidro concêntricos $(2 \mathrm{~cm}$ de diâmetro interno e altura de $25 \mathrm{~cm}$ ) com uma película promotora formada por uma tela metálica $(0,595 \mathrm{~mm})$, a qual foi cortada e adaptada rente à parede do tubo interno. A temperatura foi controlada por um banho termostático (Julabo modelo ME F25). A coluna foi alimentada a partir de um tanque de vidro de 3,0 L equipado com um agitador mecânico. A água foi bombeada para o topo da coluna por uma bomba peristáltica (Gilson, modelo minipuls 3). A concentração do ozônio na corrente gasosa foi analisada por um espectrofotômetro (Shimadzu, modelo MultiSpec1501) equipado com uma cubeta de fluxo de quartzo. A absorção máxima do ozônio é no comprimento de onda de $253,7 \mathrm{~nm}$ e sua absortividade molar $(\varepsilon)$ na fase gasosa é $2950 \mathrm{~mol} \mathrm{~L}^{-1} \mathrm{~cm}^{-1}$ (Oppenländer, 2003). O ozônio que não reage foi decomposto em solução aquosa contendo $5 \%$ de KI. A concentração de ozônio líquido foi analisada por um analisador de ozônio (Orbisphere, modelo 3600/3xx) que utiliza uma sonda polarográfica, composta de uma membrana (modelo $2956 \mathrm{~A}$ ). A sonda do equipamento de análise de ozônio no líquido tem um range de medida de 0,01 e $200 \mathrm{mg} \mathrm{L}^{-1}$. O líquido, acionado por uma bomba, irriga a membrana com vazão constante. Este dispositivo também acompanha a variação da temperatura ao longo do experimento. $\mathrm{O}$ esquema dos equipamentos utilizados está representado na Figura 1.

Os experimentos realizados nesse sistema foram realizados utilizando 1,5 $\mathrm{L}$ de água deionizada. As concentrações de ozônio no líquido e no gás foram analisadas em intervalos de $10 \mathrm{~s}$. A aquisição dos dados de $\mathrm{pH}$, temperatura e pressão do gás na entrada e saída da coluna foi realizada utilizando o programa LabView software. A temperatura da água foi mantida a $20{ }^{\circ} \mathrm{C} \pm 1{ }^{\circ} \mathrm{C}$ no tanque e monitorada na saída da coluna utilizando o equipamento Orbisphere citado acima. $\mathrm{O} \mathrm{pH}$ foi mantido constante durante todo experimento e ajustado com soluções de $\mathrm{H}_{2} \mathrm{SO}_{4}$ ou $\mathrm{NaOH}$. Um cilíndrico de oxigênio medicinal $99 \%$ foi utilizado para gerar a mistura $\mathrm{O}_{2}+\mathrm{O}_{3}$ ao passar pelo gerador de ozônio (Multivácuo, modelo MV06/200). Após a estabilização do gerador e dos valores de concentração de entrada do ozônio 
a válvula (by pass) foi aberta para o contato do gás em contracorrente com líquido bombeado do tanque pela bomba peristáltica para o topo da coluna. Experimentos foram realizados mantendo a temperatura do líquido constante a $20{ }^{\circ} \mathrm{C}$ e em valores de $\mathrm{pH} 3,5$ e 7. Utilizaram-se vazões de líquido de $0,1 \mathrm{~L} \mathrm{~min}^{-1}$ e de gás de $0,5 \mathrm{~L} \mathrm{~min}^{-1}$. As concentrações iniciais de ozônio no gás variaram de 12,4 a $31,3 \mathrm{mg} \mathrm{L}^{-1}$.

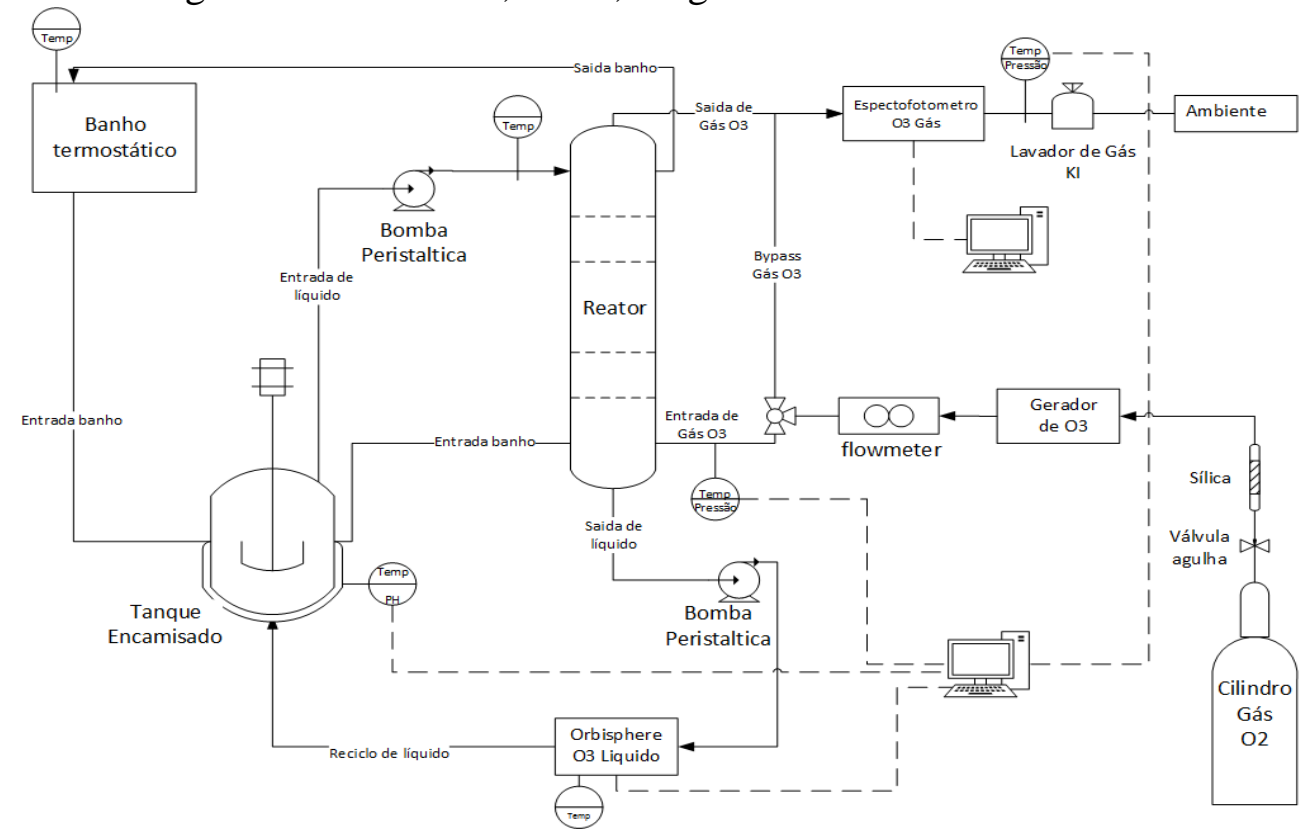

Figura 1 - Esquema do sistema utilizado.

\subsection{Metodologia para obtenção dos coeficientes}

Para obtenção do coeficiente de TM foi utilizado o balanço de massa na fase líquida que se resume na Equação 2:

$$
\frac{d\left[O_{3}\right]_{l i q}}{d t}=k L a\left(\left[O_{3}\right]_{l i q}^{*}-\left[O_{3}\right]\right)-r O_{3}(2)
$$
expressão:

Os valores de kLa foram obtidos pela integração da Equação 2 e foi obtida a seguinte

$$
\ln \frac{\left[O_{3}\right]_{l i q}^{*}-\left[O_{3}\right]_{l i q}}{\left[O_{3}\right]_{l i q}^{*}-\left[O_{3}\right]_{l i q, 0}}=-(k L a+K d) t
$$

A constante de decomposição do ozônio $(\mathrm{kd})$ foi determinada experimentalmente em função do tempo. $\mathrm{O}$ decaimento da reação foi considerado de primeira ordem e a inclinação da reta é o valor de kd.

$$
\ln \left[O_{3}\right]_{l i q, t}=\ln \left[O_{3}\right]_{l i q, 0}-k d^{*} t \quad(4)
$$

Os experimentos de decomposição foram realizados cortando o fornecimento de ozônio no sistema. A reação foi considerada de primeira ordem e a equação 4 foi usada para o cálculo experimental. A decomposição de ozônio também foi calculada seguindo o modelo proposto por Sullivan e Roth (1980). Os coeficientes foram calculados pela equação 5:

$$
k d=9.811 \times 10^{7}\left[\mathrm{OH}^{-}\right]^{0.123} \exp ^{-\frac{5606}{T}}
$$




\section{RESULTADOS E DISCUSSÃO}

Para que os parâmetros como constante de Henry e coeficiente de transferência de massa sejam calculados é necessário que as concentrações de ozônio sejam medidas tanto na corrente de gás como no líquido. Os resultados para uma corrida típica do processo de absorção de ozônio em água no sistema experimental empregado são mostrados na Figura 2.

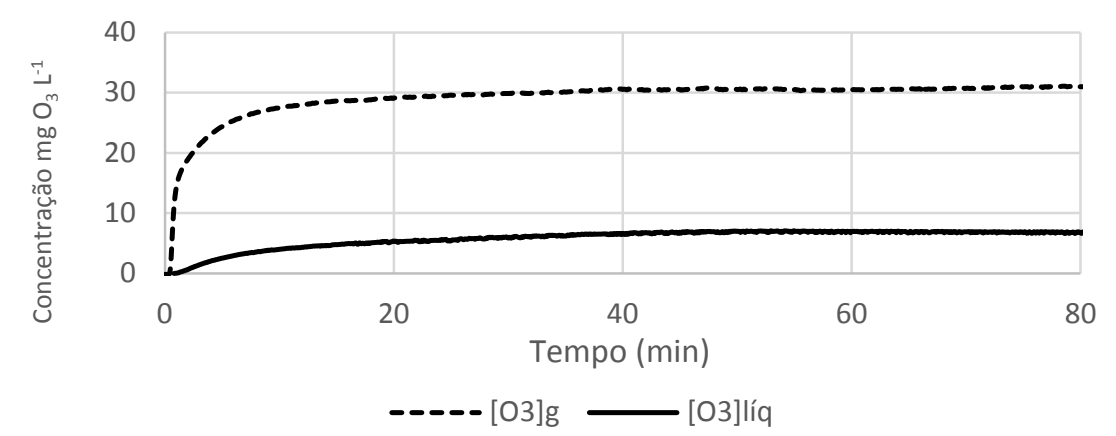

Figura 2 -Evolução típica da concentrações de ozônio no gás e no líquido em função do tempo.

A Figura 3 mostra que a concentração de ozônio dissolvido alcança o estado estacionário em aproximadamente 40 minutos após o contato do ozônio gasoso com a água na coluna. A concentração de ozônio dissolvido é afetada pelo $\mathrm{pH}$ como é relatado na literatura (Béltran, 2004; Gottschalk et al., 2000). Em concentrações reduzidas de ozônio no gás essa influência foi maior, como é observado na Figura 1. No entanto, analisando todos os gráficos em diversas concentrações observa-se que no processo de ozonização, um aumento do $\mathrm{pH}$ produz uma diminuição na concentração de ozônio no líquido, pelo aumento de íons hidróxido que reagem com o ozônio na água (Sullivan et al., 1980). Resultados semelhantes foram obtidos por Mizuno e Tsuno (2010). Tal afirmação é comprovada através dos dados obtidos na Tabela 2, na qual se verifica que em pH 3 a concentração de ozônio dissolvido no líquido chega a ser $25 \%$ maior que em $\mathrm{pH}$ 7. De acordo com Gottschalck et al. (2000) a constante adimensional de Henry é o inverso da solubilidade (s) em função da temperatura. Na Tabela 2 são mostrados os valores encontrados para constante de Henry adimensional calculados a partir da Equação 1.

Tabela 2 - Resultados encontrados para constante de Henry adimensional

\begin{tabular}{cccc|cccc|ccc}
\hline $\mathbf{p H}$ & \multicolumn{3}{c}{$\mathbf{3}$} & \multicolumn{3}{c}{$\mathbf{c}$} \\
\hline$\left[\mathbf{O}_{\mathbf{3}}\right]_{\mathbf{g}}\left(\mathbf{m g ~ L}^{-1}\right)$ & 12,39 & 24,16 & 26,8 & 13,05 & 21,79 & 30,05 & 13,86 & 22,11 & 31,30 \\
\hline$\left[\mathbf{O}_{3}\right]_{\text {líq }}\left(\mathbf{m g ~ L}^{-1}\right)$ & 2,69 & 4,58 & 6,78 & 2,15 & 4,64 & 6,29 & 2,13 & 3,48 & 5,96 \\
\hline $\mathbf{H}^{*}$ & 4,59 & 5,26 & 3,94 & 6,06 & 4,70 & 4,78 & 6,51 & 6,35 & 5,25 \\
\hline
\end{tabular}

Os valores da constante de Henry encontrados nesse trabalho foram comparados com modelos propostos pelos autores citados (Tabela 2). Tais modelos foram obtidos comparando procedimentos de diversos autores como é o caso de Bin (2006) e Morris (1988) ou obtidos experimentalmente (Langrais et al., 1991; Quederni et al., 1987). Para o cálculo do desvio nas equações em que o pH não é considerado, foi escolhido o menor valor encontrado para constante de Henry adimensional neste trabalho $(3,94)$. A escolha desse valor indica uma maior solubilidade do ozônio no líquido e menor decomposição do ozônio por meio de reações radicalares (Gottschalk et al, 2000). A partir dos desvios calculados 
observa-se que o modelo proposto por Langrais et al. (1991) é o que mais se aproxima do resultado encontrado nesse estudo, com um desvio relativo em módulo de aproximadamente $2 \%$. No entanto, nos modelos propostos na Tabela 3 ocorrem desvios de até 18,5\%, que podem ser decorrentes de diferentes métodos de análise utilizados, assim como diferenças entre os sistemas de ozonização.
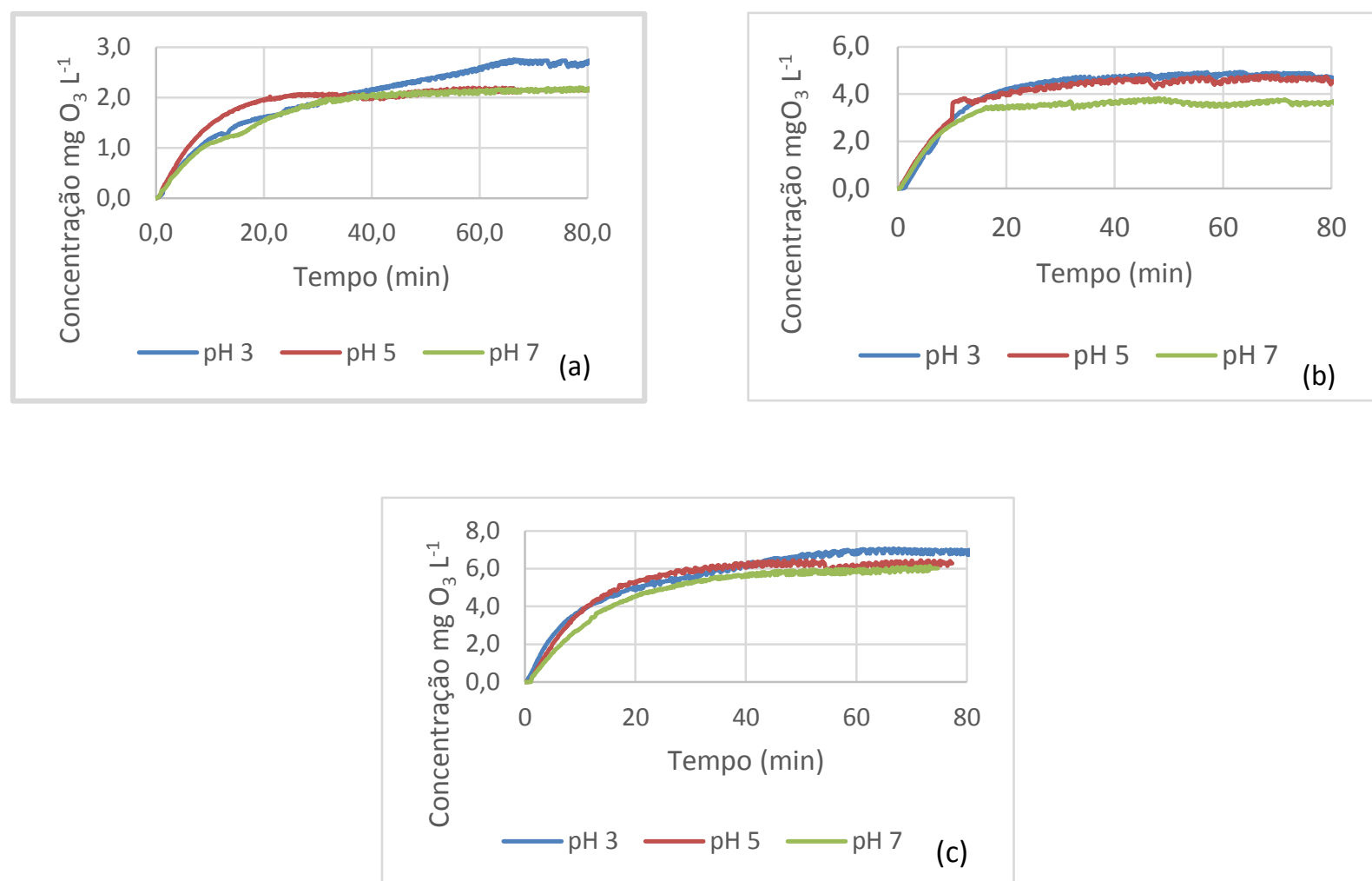

Figura 3 - Curvas de concentração de ozônio dissolvido em diferentes concentrações de ozônio no gás em relação ao pH 3, 5 e 7 (a. $\left[\mathrm{O}_{3}\right]_{\mathrm{g}}=13,1 \pm 0,7 \mathrm{mg} \mathrm{L}{ }^{-1}$; b. $\left[\mathrm{O}_{3}\right]_{\mathrm{g}}=22,7 \pm 1,3 \mathrm{mg}$ $\mathrm{L}^{-1} ;$ c. $\left.\left[\mathrm{O}_{3}\right]_{\mathrm{g}}=29,4 \pm 2,3 \mathrm{mg} \mathrm{L}^{-1}\right)$.

Tabela 3 - Desvios relativos de solubilidade em relação à literatura

\begin{tabular}{ccc}
\hline Autores & $\mathbf{H}^{*}($ adimensional) & Desvio Relativo (\%) \\
\hline Bin, 2006 & 4,16 & 5,6 \\
\hline Langlais et al., $\mathbf{1 9 9 1}$ & 3,86 & 2,0 \\
\hline Morris, 1988 & 3,21 & 18,5 \\
\hline Quederni et al., 1987 & 3,29 & 16,5 \\
\hline
\end{tabular}

Nos experimentos de Roth e Sullivan (1981) a correção do $\mathrm{pH}$ foi feita utilizado uma solução tampão de fosfato e então obtiveram uma equação em função do $\mathrm{pH}$ e da temperatura. Esses valores foram calculados e mostrados na Tabela 5. Os desvios relativos em função da temperatura de $20^{\circ} \mathrm{C}$ para o pH 3, 5 e 7 foram $35,0 \% ; 32,2 \%$ e 31,6\%, respectivamente. Os desvios foram relativamente altos, porém observa-se o mesmo comportamento dos valores calculados pela equação dos autores citados, ou seja, a constante de Henry aumentou à medida que o pH aumentou. Diferentes procedimentos de análise também podem influenciar a discrepância dos dados (Bin, 2006). Roth e Sullivan (1981) utilizaram uma técnica iodométrica modificada para analisar o ozônio dissolvido e amostras 
eram retiradas em intervalos de tempo. O sistema de análise utilizado nesse trabalho foi um sensor de ozônio dissolvido que fornece medições a cada $10 \mathrm{~s}$.

Tabela 4 - Comparação entre a constante de Henry adimensional considerando pH e temperatura

\begin{tabular}{llll}
\hline pH & $\mathbf{3}$ & $\mathbf{5}$ & $\mathbf{7}$ \\
\hline Roth et al., 1981 & 2,99 & 3,51 & 4,13 \\
\hline Neste estudo (média) & 4,60 & 5,18 & 6,04 \\
\hline
\end{tabular}

A distribuição da concentração de ozônio na fase líquida pode ser determinado pela Equação 4, estimando assim, os parâmetros que descrevem o fenômeno da transferência de massa como: o coeficiente volumétrico de transferência de massa (kLa) e o coeficiente de decomposição do ozônio (kd) (Bin, 2004). Tais parâmetros foram calculados e são mostrados na Tabela 5. O kLa é importante para a obtenção das distribuições de concentração do gás na fase líquida. Os valores de $\mathrm{kLa}$ encontrados para cada $\mathrm{pH}$ apresentaram uma variação de $30 \%$ do maior valor para o menor valor. No processo de ozonização, também é importante conhecer o efeito que produz a decomposição do ozônio na fase líquida. A decomposição do ozônio foi estudada considerando a ordem da reação de primeira ordem e os valores encontrados (Tabela 5) confirmam que a decomposição do ozônio, como esperado, aumenta ao incrementar o valor do pH (Sullivan e Roth, 1980). Uma parte da concentração do ozônio é perdida por sua decomposição na água, formando radicais livres (como radicais ${ }^{\circ} \mathrm{OH}$, entre outros) que reagem com os solutos orgânicos e com o mesmo ozônio quando é ionizado (Staehelln et al., 1982). O valor de kLa obtido na Tabela 5 é calculado a partir dos valores de $\mathrm{kd}_{\text {exp }}$ encontrados utilizando a Equação 4.

Tabela 5 - Parâmetros de transferência de massa e decomposição

\begin{tabular}{lccc}
\hline \multicolumn{1}{c}{$\mathbf{p H}$} & $\mathbf{3}$ & $\mathbf{5}$ & $\mathbf{7}$ \\
\hline $\mathbf{k L a}+\mathbf{k d}\left(\mathbf{m i n}^{-\mathbf{1}}\right)$ & $0,087 \pm 0,038$ & $0,107 \pm 0,012$ & $0,083 \pm 0,061$ \\
\hline $\mathbf{k d}\left(\mathbf{m i n}^{-1}\right)_{\mathbf{e x p}}$ & 0,011 & 0,016 & 0,019 \\
\hline $\mathbf{k d}\left(\mathbf{m i n}^{-1}\right)_{\text {lit }}$ & 0,021 & 0,052 & 0,091 \\
\hline $\mathbf{k L a}\left(\mathbf{m i n}^{-1}\right)$ & 0,076 & 0,091 & 0,064 \\
\hline
\end{tabular}

\section{CONCLUSÕES}

A absorção de ozônio foi investigada em uma coluna de parede molhada com promotor de película. Foram determinadas as constantes de Henry adimensionais, coeficientes de transferência de massa e decomposição para pH 3, 5 e 7. Os valores encontrados foram confrontados com a literatura e apresentaram um desvio relativo baixo para constante de Henry (2\%). Os valores obtidos de kd demonstraram que com o aumento do pH ocorre um aumento da taxa de decomposição do ozônio no líquido. Os valores de kLa obtidos foram 0,076; 0,091 e 0,064 $\mathrm{min}^{-1}$ valores considerados baixos se comparados a colunas de bolhas. Entretanto, foram testados para baixos valores de vazão de gás e líquido, melhores valores de kLa podem ser obtidos ajustando o sistema (mudança vazão de gás e líquido ou altura da coluna). O sistema de coluna de parede molhada com promotor de película possui a vantagem de fácil montagem, além da diferença em relação à maioria dos sistemas de ozonização em que é necessário estudar o comportamento das bolhas. A utilização do 
promotor de película promove o aumento da área de contato entre o gás e o líquido formando um filme uniforme.

\section{REFERÊNCIAS}

BELTRÁN, F. J. Ozone reaction kinetics for water and wastewater systems. Valência: Lewis Publishers, p. 358, 2004.

BIN, A. K. Ozone dissolution in aqueous systems treatment of the experimental data. Exp. Therm. Fluid. Sci., v.28, n.5, p.395-405, 2004.

BIN, A. K. Ozone solubility in Liquids. Ozone: Sci. and Eng., p. 65-75, (2006).

CREMASCO, M. A. Fundamentos de transferência de massa. $2^{\mathrm{a}}$ ed. Campinas: Editora da Unicamp, p. 729, 2002.

CULLEN, P. J.; TIWARI, E. K.; MUTHUKUMARAPPAN, K. Modeling approaches to ozone processing of liquid foods. T. Food Sci. Technol., v.20, n.3-4, p.125-136, 2009.

DANCKWERTS, P.V. Gas-liquid Reactions, McGraw-Hill, New York, 1970.

GOTTSCHALK, C.; LIBRA, J.A.; SAUPE, A. Ozonation of Water and Waste Water, 1st Ed.; Wiley-VCH: Weinheim, Germany,2000.

LANGLAIS, B.; RECKHOW, D. A.; BRINK, D. R. Ozone in Water Treatment: Application and Engineering. AWWA Research Foundation, Chelsea, Michigan: Lewis Publishers Inc., 1991.

MIZUNO, T.; TSUNO, H. Evaluation of solubility and the gas-liquid equilibrium coefficient of high concentration gaseous ozone to water. Ozone: Sci. and Eng., v.32, n.1, p.3-15, 2010.

MORRIS, J. C. The aqueous solubility of ozone - A review. Ozone news, ${ }^{\circ}$ 1, p. 14-16, (1988).

OPPENLÄNDER, T. Photochemical Purification of Water and Air, 1st Ed.; Wiley-VCH: Weinheim, Germany, 2003.

QUEDERNI, A.; MORA, J. C.; B, R. S. Ozone absorption in water: mass transfer and solubility. Ozone: Sci. and Eng., v.9, n.1, p.1-12, 1987.

REINIK, J.; JAKOBSSON, K.; KALLAS, J. 2,4-Xylidine Degradation with Ozonation: Mass Transfer and Reaction Kinectics. Ozone: Sci. and Eng., v.26, p.499-509, 2004.

RIZZUTI, L.; AUGUGLIARIO, V.; MARRUCCI, G. Ozone absorption in alkaline solutions. Chem. Eng. Sci., v.31, n.6, p.877-880, 1976.

RODRIGUEZ-FLORES, H.A.; MELLO, L.C.; SALVAGNINI, W.; PAIVA, J.L. Absorption of $\mathrm{CO}_{2}$ into aqueous solutions of MEA and AMP in a wetted wall column with film promoter. Chem. Eng. Process., v. 73, p.1-6, 2013.

ROTH, J. A; SUlLIVAN, D. E. Solubility of ozone in water. Ind. Eng. Chem. Fundam., v.20, n.2, p.137-140, 1981.

SALVAGNINI, W.; TAQUEDA, M.A. A falling-film evaporator with film promoters. Ind. Eng. Chem. Res., v. 43, p. 6832-6835, 2004.

SOTELO, J. L.; BELTRAN, J. F.; BENITEZ, F. J.; HEREDIA, J. Ozone decomposition in water: kinetic study. Ind. Eng. Chem. Res., v.26, n.1, p.39-43, 1987.

STAEHELLN, J.; HOLNE, J. Decomposition of ozone in water: rate of initiation by hydroxide ions and hydrogen peroxide. Environ. Scl. Technol, v.16, n.10, p.676- 681, 1982.

SULLIVAN, D. E.; ROTH, J. A. Kinetics of ozone self-decomposition in aqueous solution. AIChE Symp. Ser-Water, v.197, n.76 p.142-149, 1980. 